\title{
Análisis de la problemática del agua en Paraguay
}

\section{Analysis of the water problem in Paraguay}

*Salas Dueñas DA

\author{
Fundación Moisés Bertoni, Asunción, Paraguay
}

\section{RESUMEN}

La situación de mediterraneidad en el semi-trópico del Paraguay lo hace especialmente susceptible a la problemática ligada con el agua. Es por esto, que comprender y entender las limitaciones que el país tiene al respecto debe ser uno de los primeros pasos que determinen el modelo de desarrollo que se quiere como nación. Del mismo modo, contar con un análisis regionalizado de las principales fuentes de impacto así como de los efectos de las mismas, debería facilitar la toma de decisiones correctivas.

Palabras clave: Paraguay, agua, problemática, humedales, acuíferos, sectorización.

\section{ABSTRACT}

The situation of landlocking of the semi-tropics of Paraguay makes it especially susceptible to problems linked to water. Therefore, understanding and comprehending the limitations of the country related to water access should be one of the first steps to determine the development model required as a nation. In the same way, having a regionalized analysis of the major impact sources and their effects should facilitate making corrective decisions.

Keywords: Paraguay, water, problem, wetlands, aquifers, sectorization.

\section{Análisis de la problemática del agua en Paraguay}

Paraguay es un país mediterráneo ubicado en el centro de Sur América cuyo territorio ocupa una superficie de $406.752 \mathrm{Km} 2$. El Río Paraguay, perteneciente a la Cuenca del Plata, divide al país en dos regiones naturales. La región Oriental es una llanura interrumpida por colinas y cordilleras bajas en donde se asienta gran parte de la población. La región Occidental es una gran Ilanura aluvial con un ligero declive hacia el Río Paraguay (1).

Los problemas ambientales relacionados con los recursos hídricos en el Paraguay son efectos de la erosión provocada por la deforestación de las cuencas, la introducción de pesticidas, la contaminación por desechos domésticos e industriales y los efectos ambientales de los embalses hidroeléctricos (2).

Si bien en la región oriental existen abundantes fuentes de agua (arroyos, ríos, humedales) y vertientes naturales (Ykuà) con potencial para surtir a buena parte de la población, uno de los principales problemas del sector rural es la falta de disponibilidad de agua potable, ya que muchas de estas fuentes e inclusive napas freáticas superficiales, se hallan contaminadas o se ha privatizado el acceso a las mismas.

En la región de Chaco, el río Paraguay y el río Pilcomayo, que bordean dicha región, son los únicos cuerpos de agua que tienen agua todo el año, ya que muchos de los cauces y fuentes son solamente de carácter temporal (3).

La principal fuente de agua en esta región es subterránea, sin embargo, la presencia de sales entre los sedimentos de la mayoría de los nacientes limita seriamente su utilización. Adicionalmente, esta agua fue depositada en épocas glaciales y no recibe ningún tipo de

*Autor Correspondiente: Danilo A. Salas Dueñas, Fundación Moisés Bertoni, Asunción, Paraguay Email: DaniloSalas@gmail.com

Fecha de recepción: julio 2014; Fecha de aceptación: diciembre 2014 
recarga, por lo que su disposición es limitada y muchas de estas fuentes están expuestas a contaminación por aguas residuales domésticas e industriales (4).

Desde la perspectiva del transporte y las comunicaciones, cabe recordar que la condición de país mediterráneo hace que buena parte de su inserción en el ámbito internacional sea, hasta mediados de los años 60, resultado de su capacidad de navegación fluvial. El río Paraguay que recorre el país de norte a sur y el río Paraná que le bordea por el este y el sur, han sido fundamentales para estas actividades (5).

En el caso del sector energético, es más que evidente el potencial energético a partir de los recursos hidroeléctricos. En los años 60, se construyó la represa de Acaray y en los 70 se inició conjuntamente con Brasil la construcción de Itaipú, conocida como la planta hidroeléctrica más grande del mundo. En la misma década, se inició conjuntamente con Argentina el trabajo para la construcción de la represa de Yacyretá. Igualmente, desde entonces también se habla de la construcción de otra represa conjunta con Argentina denominada Corpus, y ahora de Ytacurubí, ambas sobre el río Paraná (6).

Considerando los aspectos de servicios de agua potable y saneamiento, el estado tiene dos entidades públicas. "ESSAP" que es una entidad autárquica del estado y sirve a comunidades urbanas de más de 4.000 habitantes, mientras que "DIGESA" una entidad dependiente del Ministerio de Salud, se hace cargo de las poblaciones menores y del sector rural. Sin embargo, su grado de cobertura es mínimo, lo que constituye un riesgo para la salud pública (7).

Un verdadero diagnóstico de la problemática requiere profundización y complementación con los diferentes estudios que se desarrollan en función de las instituciones, de acuerdo con sus funciones, competencias y programas.

\section{Las condiciones de Paraguay}

\section{La oferta de agua dulce}

Paraguay por su ubicación geográfica y sus condiciones de mediterraniedad tiene una precipitación media anual de $93 \mathrm{~mm}$ (según los datos de 22 estaciones a lo largo del país), lo que representa un déficit de recursos hídricos, si se compara con el promedio mundial de precipitación, que se encuentra alrededor de los $900 \mathrm{~mm}$ y con el de Sudamérica, que está cerca de los $1.600 \mathrm{~mm}$ (1).

Como se puede deducir de los anteriores datos, el caudal específico de escorrentía superficial en Paraguay es muy bajo. Sin embargo, la oferta de agua es superior debido a la presencia de muchos pequeños cuerpos de agua que proporcionan sus recursos a comunidades muy puntuales y a la existencia de una gran reserva de agua subterránea y de grandes cuencas internacionales que recorren al país.

Adicionalmente, la red de estaciones no es suficiente para proporcionar datos más ajustados a la realidad.

\section{Precipitación}

Debido a las características de la circulación atmosférica y a las diferencias en el contenido de humedad, en el territorio nacional se presenta un régimen pluviométrico muy variado. Mientras que en el Chaco se registran los promedios anuales más bajos, cercanos a $55 \mathrm{~mm}$, en algunos lugares de la región Oriental los valores de precipitación son los más altos del país con $135 \mathrm{~mm}$ de promedio.

La distribución de la precipitación en el territorio está determinada por las variaciones espacio-temporales de los sistemas de circulación general de la atmósfera de la subtropical y por la interacción de estos factores con las características fisiográficas del país (1). 


\section{Los Humedales del Paraguay}

Paraguay presenta una extensa área de ambientes húmedos, conocidos como pantanales, selvas de ribera, embalsados, esteros y otras tantas denominaciones que tienen en común ser considerados humedales por la convención Ramsar.

Las áreas de humedales ocupan aproximadamente un área que equivale entre un $30 \%$ a $40 \%$ (8), aunque se cuenta con registros anteriores de al menos un $26 \%$ de la superficie del territorio paraguayo (9) lo que representa un área que puede variar entre los 100.000 $\mathrm{Km}^{2}$ y los $150.000 \mathrm{Km}^{2}$, respectivamente.

Algunos de los humedales del Paraguay, hacen parte de El Pantanal, que es considerado el mayor humedal del mundo, con unos $140,000 \mathrm{Km}^{2}$ y cubre áreas en Brasil, Bolivia, y Paraguay. Forma las nacientes del Río Paraguay, considerado uno de los más importantes reservorios de agua para los países de la región. Es el mayor reservorio de plantas acuáticas del hemisferio occidental y contiene grandes concentraciones de peces y aves, lo que posiblemente le confiere el contener la mayor concentración de especies acuáticas del mundo (5).

Estos humedales se encuentran distribuidos en las dos regiones naturales del país. La Occidental o Chaco, donde existen extensas áreas con suelos formados básicamente por arcillas y limos, los cuales no permiten la filtración del agua, facilitando la formación de extensos pantanales (10), y la formación de un grupo de importantes biomas, entre los que sobresalen los llamados Palmares, caracterizados por un estrato arbóreo de la palmera Karanda 'y (Copernicia alba). Gran parte de ellos sufre inundaciones periódicas y son un importante refugio para un gran número de aves acuáticas. Este bioma está en peligro debido a la expansión de la frontera agrícola y las quemas periódicas que se realizan de los mismos.

Otro bioma de gran importancia es el denominado Espartillar, el cual generalmente surge en áreas de paleocauces. El estrato herbáceo ocupa un $90 \%$ dominado por el Espartillo (Elionurus muticus) y unos estratos arbustivos y arbóreos muy abiertos. En este último, algunas especies alcanzan grandes tamaños. Su principal amenaza proviene de la ocupación por actividades agrícolas (5).

De la misma forma existen algunos otros tipos de bioma, como los Bañados, que es una formación de sabana, y los Médanos, que son dunas de arena con grave peligro de desertificación, por destrucción de la vegetación que las cubre.

Se considera que los humedales del chaco paraguayo son producto de la interacción de las aguas de desborde del Río Paraguay al este, las inundaciones provenientes del río Pilcomayo al sureste y las lluvias ocurridas al este de la región (11).

\section{Agua subterránea}

El volumen de agua subterránea con que cuenta el país no ha sido cuantificado en su verdadera magnitud, configurándose como un capital complementario al capital hídrico superficial. Este potencial debe ser debidamente estudiado, valorado cualitativa y cuantitativamente, ya que constituye una oferta alternativa, que, en muchas áreas, ya está siendo utilizada sin planificación ni manejo.

La principal fuente de agua subterránea es el Acuífero Guaraní, nombre dado en honor al pueblo indígena Guaraní, considerado uno de los más grandes reservorios del mundo. Está localizado en jurisdicción de cuatro países del Mercosur: Argentina, Brasil, Paraguay y Uruguay.

El sistema del Acuífero Guaraní, tienen una superficie total estimada de aproximadamente 1,2 millones de $\mathrm{km}^{2}$, de los cuales unos $71,700 \mathrm{~km}^{2}$ corresponderían a territorio paraguayo y se estima que en total unas 15 millones de personas viven en su área de influencia. 
Cerca de $40,000 \mathrm{~km}^{3}$ de agua son contenidos por el acuífero y se estima que cerca del $90 \%$ de la misma es potable. Sin embargo, la potabilidad en el nivel local puede ser reducida por problemas de salinización. El acceso al mismo varía entre una profundidad de 250 metros hasta 1.000 metros en algunos otros lugares (12).

Adicionalmente a la buena calidad del agua, ya que cerca del $90 \%$ de la misma puede ser consumida, se destaca la calidad térmica de la misma, al aflorar en algunos lugares con temperaturas entre los $33^{\circ} \mathrm{C}$ y $\operatorname{los} 50^{\circ} \mathrm{C}$, lo que adicionalmente al potencial turístico le otorga un potencial como fuente de energía alternativa.

Sin embargo, la creciente demanda por agua en algunas regiones tanto por crecimiento demográfico como por expansión económica, y la mala utilización de productos agrotóxicos pone en riesgo el potencial que presenta actualmente dicha reserva de agua.

Si bien los niveles de contaminación en el Paraguay son mínimos, no se debe dejar de lado el problema que se viene generando por la mala utilización de las tecnologías en la perforación de pozos en algunas zonas, y el que los graves problemas de contaminación en lugares lejanos de Brasil y Argentina, debido a la dinámica del agua, afectan la totalidad de la región.

Si recordamos los problemas de déficit de agua en un amplio sector del Paraguay, todo lo anterior detalla lo estratégico que es dicho reservorio para Paraguay y para la región en general.

\section{Balance hídrico}

A través del balance hídrico se pueden caracterizar las diferentes regiones y establecer dentro de qué rango de disponibilidad natural del recurso se encuentran éstas. El balance muestra el resultado de la interrelación de los parámetros hidrológicos y meteorológicos (precipitación, evapotranspiración potencial, evapotranspiración real y escorrentía) caracterizando los ambientes de las regiones. Sin embargo, a pesar de no contar con los datos necesarios para la obtención del mismo podemos decir con buen margen de certeza que va desde el árido y seco en el Chaco hasta el muy húmedo en zonas de Ñeembucú.

\section{La problemática hídrica nacional}

Se presentan situaciones de dificultad en la disponibilidad espacial y temporal del agua superficial en el nivel regional y local, causadas por diferentes aspectos tales como:

-Demanda de agua en zonas donde la oferta hídrica es limitada.

-El mal uso dado históricamente a algunos cuerpos de agua alterando la oferta hídrica natural en cantidad y en distribución espacio-temporal.

-Deterioro de la calidad del agua por sedimentos y contaminación. (Ver Tablas 1 y 2)

Adicionalmente, fenómenos climáticos de los últimos años, denominados ciclos del Niño y la Niña han agravado dicha problemática. 
Tabla 1. Sectorización de la Problemática del agua en Paraguay en la Región Oriental.

\begin{tabular}{|c|c|c|c|}
\hline Actividad & & Efectos & Área \\
\hline \multirow{6}{*}{$\begin{array}{l}\text { Centro } \\
\text { Oeste }\end{array}$} & Urbana e industrial & $\begin{array}{l}\text { Contaminación superficial y } \\
\text { acuíferos }\end{array}$ & \multirow{6}{*}{$\begin{array}{l}\text { REMA (Región Metropolitana } \\
\text { de Asunción) } \\
\text { Cuenca de Ypacarai. } \\
\text { REMA } \\
\text { Cuenca de Ypacarai } \\
\text { Cuenca del Ypoa } \\
\text { REMA } \\
\text { REMA } \\
\text { Villa Hayes } \\
\text { Todos los Deptos. }\end{array}$} \\
\hline & Mataderos y frigoríficos & Aguas superficiales & \\
\hline & $\begin{array}{l}\text { Expansión Úrbana } \\
\text { desordenada }\end{array}$ & Destrucción de fuentes de agua & \\
\hline & Cementera & Aguas superficiales & \\
\hline & Metalúrgica & $\begin{array}{l}\text { Contaminación superficial y } \\
\text { acuíferos }\end{array}$ & \\
\hline & Prácticas agrícolas & $\begin{array}{l}\text { Colmatación por erosión y } \\
\text { contaminación por pesticidas }\end{array}$ & \\
\hline Sur & Represas & $\begin{array}{l}\text { Eutrofización } \\
\text { Aumento de vectores }\end{array}$ & Itapúa -Misiones \\
\hline \multirow[b]{2}{*}{ Sur Este } & Industria maderera & \multirow{2}{*}{$\begin{array}{l}\text { Deforestación de cuencas } \\
\text { Conflictos por uso del uso agua } \\
\text { Contaminación por agroquímicos }\end{array}$} & Itapúa - Caazapá \\
\hline & $\begin{array}{l}\text { Proyectos agrícolas con } \\
\text { irrigación }\end{array}$ & & Itapúa - Misiones \\
\hline Sur Oeste & $\begin{array}{l}\text { Industria Pesquera } \\
\text { Proyectos agrícolas y } \\
\text { ganaderos con irrigación }\end{array}$ & $\begin{array}{l}\text { Drenaje de cuencas y humedales } \\
\text { Conflictos por uso del uso agua } \\
\text { רContaminación por agroquímicos }\end{array}$ & $\begin{array}{l}\text { S̃̃eembucú - Itapuá - } \\
\text { Misiones }\end{array}$ \\
\hline Centro & Industria azucarera & $\begin{array}{l}\text { Contaminación de agua } \\
\text { superficial y subterránea }\end{array}$ & Guairá - Caaguazú \\
\hline Norte & $\begin{array}{l}\text { Agricultura extensiva } \\
\text { Ganadería } \\
\text { Industria pesquera }\end{array}$ & $\begin{array}{l}\text { Erosión } \\
\text { Colmatación } \\
\text { Contaminación } \\
\text { Sobreexplotación pesquera }\end{array}$ & $\begin{array}{l}\text { Amambay - Canindeyú - } \\
\text { Concepción - San Pedro }\end{array}$ \\
\hline
\end{tabular}

Tabla 2. Sectorización de la Problemática del agua en Paraguay en la Región Occcidental (Chaco).

\begin{tabular}{|c|c|c|c|}
\hline Actividad & & Efectos & Área \\
\hline \multirow{2}{*}{ Chaco Central } & $\begin{array}{l}\text { Mataderos } \\
\text { Industria lechera }\end{array}$ & $\begin{array}{l}\text { Contaminación orgánica e } \\
\text { inorgánica de acuíferos }\end{array}$ & Colonias Mennonitas \\
\hline & Agricultura & $\begin{array}{l}\text { Sequías estacionales } \\
\text { Salinización de acuíferos }\end{array}$ & $\begin{array}{l}\text { Boquerón -Presidente } \\
\text { Hayes }\end{array}$ \\
\hline $\begin{array}{l}\text { Noreste del } \\
\text { Chaco }\end{array}$ & $\begin{array}{l}\text { Ganadería extensiva } \\
\text { Minería en Brasil } \\
\text { Industria Pesquera }\end{array}$ & $\begin{array}{l}\text { Contaminación de cuerpos de agua } \\
\text { por agroquímicos } \\
\text { Contaminación con mercurio } \\
\text { Sobreexplotación }\end{array}$ & Alto Paraguay \\
\hline $\begin{array}{l}\text { Noreste y } \\
\text { centro oeste } \\
\text { del Chaco }\end{array}$ & $\begin{array}{l}\text { Minería en Bolivia } \\
\text { Deficiente gestión del } \\
\text { Pilcomayo }\end{array}$ & $\begin{array}{l}\text { Contaminación por erosión } \\
\text { Conflictos por uso del agua }\end{array}$ & $\begin{array}{l}\text { Boquerón - } \\
\text { Presidente Hayes }\end{array}$ \\
\hline
\end{tabular}




\section{Causas Subyacentes de la problemática}

Algunas de las causas subyacentes en la problemática del agua, referidas a los aspectos institucionales y sociales pueden ser señaladas como:

- La naturaleza de la inequidad en el acceso al agua ha generado tasas de extracción muy superiores a las socialmente deseables y naturalmente sustentables.

- A pesar de la variedad de instrumentos económicos, administrativos y de inversión, entre otros, que pueden ser utilizados para lograr el buen manejo del recurso hídrico, se ha dejado toda la responsabilidad a instrumentos jurídicos, los cuales a su vez no han sido aplicados o en su defecto han sido mal aplicados, no permitiendo sancionar por acciones que conllevan el deterioro ambiental por mal uso del agua, o las fuentes de la misma y/o vertimientos.

- La situación económica y sociocultural de gran parte de la población paraguaya la induce a consumir intensamente los recursos naturales y no le permite acceder fácilmente a tecnologías con las que podría mejorar su ingreso sin deteriorar el medio natural, en este caso, el recurso hídrico.

- Los procesos de planificación territorial azarosa, en las que se han degradado áreas potencialmente de interés hídrico.

- Los patrones de producción y consumo caracterizados por un uso ineficiente del recurso hídrico.

- La débil respuesta de las instituciones del estado para afrontar y orientar soluciones concretas a los problemas ambientales.

- La reducida investigación que ha redundado en una base científica y un sistema de información insuficiente.

- La falta de tecnología apropiada para el uso y conservación del agua.

- En la región Oriental, la conducta alrededor del agua ha obedecido a una cultura de abundancia del recurso, mientras que en la región Occidental se afrontan problemas de escasez, hecho que no ha facilitado generar una cultura social del agua.

- Deficiencias en el diseño y aplicación de los modelos de administración y conservación del agua y dificultades para poner en funcionamiento las pautas de la descentralización, desconociendo el concepto de manejo de cuencas, lo que sobrepasa los límites políticos y jurisdiccionales.

- Inadvertencia del agua como componente regulador del sistema natural. Ha faltado la aplicación de la concepción de un manejo integral de los recursos naturales sueloagua-vegetación.

\section{CONCLUSIÓN}

Existe una errada percepción social sobre el tema de disponibilidad y calidad de los recursos hídricos, en cuanto a lo inagotable de los mismos, en algunos casos generando recientes conflictos sociales por recursos. Todo este panorama va de la mano con diversos problemas estructurales claves para poder determinar el verdadero estado del recurso en Paraguay. La deficiencia en información, la falta de políticas claras, la multiplicidad de instancias públicas, la poca operatividad de entes de control y regulación, entre otras, no solo son un factor multiplicador de la problemática, sino que permiten señalar que existen situaciones que deben ser modificadas para remediar los diversos problemas crecientes en relación con este recurso, pero sobretodo son un llamado de alerta a los tomadores de decisiones de la urgente necesidad de tomar cartas en el asunto en busca de una solución real y duradera. 


\section{REFERENCIAS BIBLIOGRÁFICAS}

1. Acevedo C, Fox J, Gauto R, Granizo T, Kell S, Pinazzo J, et al. Áreas Prioritarias para la conservación en la Región Oriental del Paraguay. Centro de Datos para la Conservación. Ministerio de Agricultura y Ganadería. Asunción: Graphis; 1990.

2. Fundación Moisés Bertoni. Subprograma de Humedales. Documento de trabajo. Asunción: Fundación Moisés Bertoni; 2001.

3. Bucher E, Bonnetto A, Caneveri P, Castro G, Hunszar P, Stone T. Hidrovía, un examen ambiental de la vía fluvial Paraguay - Paraná. Monomet, Massachusttes: Wetlands for the Americas; 1993.

4. Ministerio de Agricultura y Ganadería, Dermasur. Proyecto de desarrollo rural y mejoramiento ambiental de la región sur de Pilar: Documento informativo. Pilar: Ministerio de Agricultura y Ganadería, Agencia de Cooperación Internacional del Japón; 1997.

5. Willink PW, Chernoff B, Alonso LE, Montambault JR, Lourival R, editors. Conservation priorities: The role of RAP. A Biological assessment of the aquatic ecosystems of the Pantanal, Mato Grosso do Sul, Brasil /Internet/. Washington: Conservation International; 2000. /citado 20 feb 2015/.

Disponible en: https://library.conservation.org/Published\%20Documents/2009/RAP_Bulletin_18.pdf

6. Tarak $P$, direc proyecto, Grupo Y'Guazú. Bases para la armonización de exigencias ambientales en el Mercosur. Buenos Aires: Fundación Ambiente y Recursos Naturales; 1995.

7. Molinas M, Escobar M. Derecho a un ambiente sano. En: Informe sobre los derechos humanos en Paraguay año 1996. Asunción: SERPAJ; 1996.

8. Salas D, Mereles F, Yanosky A, editores. Los humedales de Paraguay. Asunción: Comité Nacional de Humedales; 2004.

9. Mereles F, Degen R, López N. Humedales en el Paraguay: Breve reseña de su vegetación. Amazonia. 1992; 12(2):305-16.

10. Vera V, Camé O, Barboza F, Ortíz R, Terol G, Fracchia F, et al. Iniciativas transfronterizas de conservación en el Chaco Paraguayo: Plan de acción de conservación 2000-2004. Asunción: TNC, USAID; 2000.

11. Fernández Reyes L, Moura DM, editores. Humedales de Iberoamérica: Experiencias de estudio y gestión. CYTED, Programa Iberoamericano de Ciencia y Tecnología para el Desarrollo, Red Iberoamericana de Humedales. La Haban: CYTED; 2005.

12. Facetti JF. Estado ambiental del Paraguay: presente y futuro. Asunción: GTZ, Secretaría del Ambiente; 2002. 